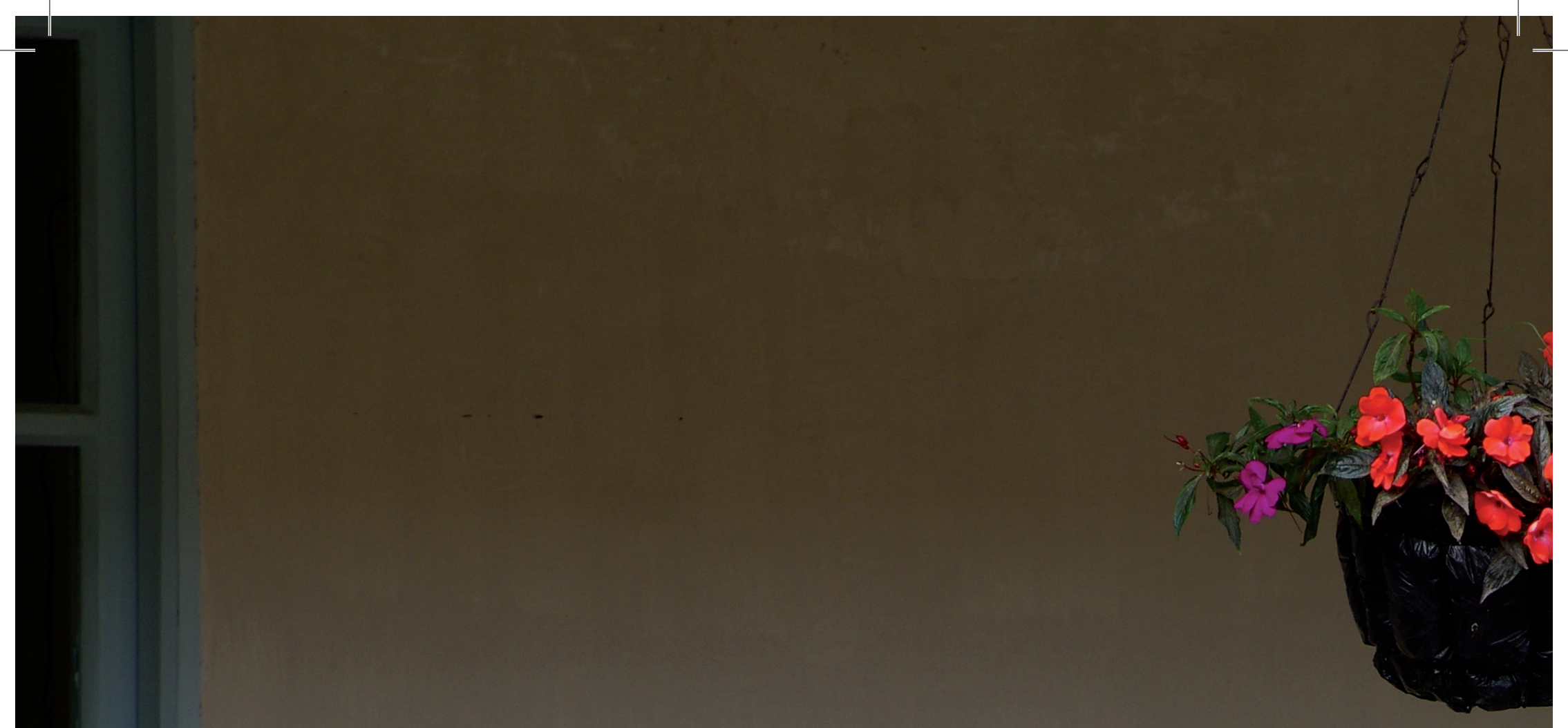




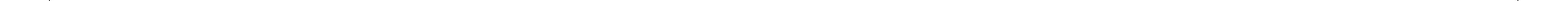




\section{Bandolarium, cuatro obras para bandola sola}

\section{Resumen}

Se busca comprender el papel de la bandola andina colombiana en su contexto contemporáneo e histórico, proponiendo la interacción de este instrumento popular con los retos y circunstancias de la música global y más concretamente de la música académica. Para ello, el presente artículo aporta nuevas formas de interpretación de la bandola andina desde lo que se concibe como técnicas extendidas y complementarias, las cuales van más allá de las técnicas tradicionales de ejecución del instrumento y logran un desarrollo diferente del músicobandolista haciéndolo más integral en términos musicales e interpretativos.

Palabras clave: Bandola andina colombiana, técnicas extendidas y complementarias, bandola sola.

\section{BANDOLARIUM, FOUR SOLO PIECES FOR BANDOLA}

Abstract: This isan effort to understand the role played by the bandola -a Colombian musical instrument from the Andes- within a contemporary context, so as to foresee the ways in which this instrument may interact with the challenges and circumstances of global and particularly academic music, without losing its folkloric essence. So, this paper proposes new ways of playing the bandola from the perspective of what is known as "extended and complementary techniques", which go far beyond traditional performing procedures, allowing the bandolist musicians to achieve a different and more holistic development of their performing and musical skills.

Keywords: Colombian bandola of the Andes, extended techniques, complementary techniques, bandola solo.

\section{Bandolarium, 4 obras para bandola "sola"}

Resumo: Procura-se compreender a função da bandola andina colombiana desde um contexto contemporâneo e histórico, que permita interatuar desde a sua essência de instrumento popular com os retos e circunstancias da musica global e mais especificamente da musica acadêmica, para isso o presente artigo aporta novas formas de interpretação da bandola andina desde o que se concebe como "técnicas estendidas e complementares", as quais vão alem das técnicas tradicionais de interpretação do instrumento e conseguem um desenvolvimento diferente do musico - bandolista fazendo ele mais integral em termos musicais e interpretativos.

Palavras chave: bandola andina colombiana, técnicas estendidas e complementares, bandola "sola". 
Desde la institucionalización de la cátedra de bandola en Colombia, específicamente en Bogotá, el instrumento ha tenido un proceso de formación corto en comparación con otros instrumentos académicos, es por eso que no goza de mucho reconocimiento en el ámbito de la composición. El repertorio académico utilizado para este fin se remite a instrumentos análogos (mandolina, bandurria, cavaquiño, solo por mencionar algunos) o, en varios casos, adaptaciones de otros instrumentos. Las composiciones idiomáticas para bandola se resumen en 24 estudios compuestos por el maestro Fabián Forero Valderrama, quien estudio en Europa técnicas en instrumentos de plectro, estos estudios son la única obra publicada para este fin, está dividida en dos tomos (12 estudios latinoamericanos para bandola solista y 12 estudios caprichos para bandola) y en ella se plantean recursos técnicos interesantes para el desarrollo, composición y ejecución de cada pieza. La utilización de ritmos o temas latinoamericanos en el primer libro y un depurado trabajo en el segundo ${ }^{1}$ enriquecen el tratamiento polifónico de texturas con el uso de aspectos técnicos nuevos y pensados para el instrumento, así como "prestados de otros" (dobles cuerdas, melodía con acompañamiento en acordes, textura a dos y tres partes, glissando, staccato, armónicos artificiales, alza púa, contra púa, ligados, media ceja, uso del dedo anular, etc.).

Las propuestas musicales que abordan temáticas de composición idiomática son creadas mediante una tradición de muchos años, e incluso siglos, pasando por estilos, culturas, épocas y hasta modificaciones morfológicas de estos instrumentos. La bandola, siendo un instrumento tan joven en cuanto a su construcción, utilización, y modificación morfológica, aun en la música utilizada (música andina colombiana la cual no tiene más de 150 años), brinda, con respecto a su ejecución, un marco referencial limitado, pues por tradición se le han asignado unos roles melódico y rítmico definidos, se ha utilizado en unas músicas características, e incluso, se le ha dado cierto estatus social. No obstante, Las músicas tradicionales alimentaron el repertorio para el instrumento y fueron el motor para que estudiosos y músicos académicos se interesaran en el desarrollo del mismo; la creación de festivales, encuentros, talleres, conciertos, etc. dieron un nuevo aire a la música andina colombiana y con ello a la bandola, pero a su vez la parte técnica y de desarrollo individual también tomaron un rumbo más serio y claro.

Los programas de música donde la bandola se enseña como instrumento principal han creado una generación de intérpretes con nuevas ópticas hacia el instrumento,

\footnotetext{
${ }^{1}$ El primer libro del maestro Fabián Forero (12 estudios latinoamericanos para bandola colombiana) es la mirada desde el virtuoso, ya que se plantean aspectos de un altísimo nivel de dificultad, nunca antes intentados en el instrumento. El segundo libro (Arte y ejecución de la bandola andina colombiana), aborda objetivos más técnico-pedagógicos para el desarrollo del naciente bandolista-solista.
}

entre ellas la bandola solista ${ }^{2}$ y la bandola sola. ${ }^{3}$ Este concepto busca la inclusión de nuevas músicas, nuevos ensambles y nuevas formas de composición idiomática, ya no como una idea ajena, sino como una construcción colectiva dentro del medio académico y de concierto. Es por eso que la composición idiomática para la bandola, debe basarse en el conocimiento técnico-expresivo del instrumento y las músicas que se quieren interpretar en ella (música académica, popular, contemporánea, comercial).La creación de obras con este tipo de características: "sola" y "solista", son importantes para el desarrollo del instrumento y su consolidación tanto en el medio académico como en el popular.

\section{Mandolina y bandurria, abuelos y ancestros}

"La mandolina de forma semejante a la mandora, apareció en el siglo XVII y se toca con plectro de pluma, adquirió nacionalidad y estilo italianos" (Marulanda y Gonzáles, 1994) La mandolina es uno de los instrumentos de plectro más conocidos en el mundo, su repertorio abarca diferentes periodos de la historia de la música occidental; transcripciones y adaptaciones de estas obras se han retomado para la bandola, fortaleciendo procesos de enseñanza en universidades y academias:"estudiar los diferentes estilos del repertorio de época (mandolina) fortalece la bandola tanto es su técnica, su interpretación y su institucionalización, ya que la bandola pasa de ser un instrumento nacional a un instrumento de nivel internacional" (Forero, 2011, clase colectiva, UPN). En los últimos años la bandola ha interpretado los conciertos para mandolina de Antonio Vivaldi, así como sonatinas para piano y mandolina de compositores como Ludwig van Beethoven, Giovanni Battista entre otros, algunas de estas obras han sido obligatorias para recitales y exámenes de grado en distintas universidades y programas de música.

"Desde el siglo XVIII la bandurria se afina a la quinta, y posee cinco órdenes de cuerdas dobles, afinadas al unísono: después se le añadió una sexta cuerda doble, en el registro del sol" (Marulanda y Gonzáles, 1994). La bandurria es el instrumento más parecido a la bandola andina colombiana, ya que su manera de afinación es por intervalos de cuartas (igual que la bandola). Los repertorios de la bandurria son estudiados dentro de los programas de música para bandola, al tener la

2Un instrumento "solista" puede encontrarse acompañado
de otro instrumento siempre y cuando no pierda su papel de
solista, algunos ejemplos están en los conciertos para solista
y orquesta, suites, sonatas y demás obras de cámara para
solista y acompañante, como también las partes solistas en
obras sinfónicas (y/o) de cámara.
3 Un instrumento "solo" puede interpretar obras sin la ayuda
de otros instrumentos ya sean solistas o acompañantes,
algunos ejemplos son las partitas para violín solo de J. S.
Bach, así como de otros compositores, sonatas, suites para
piano, guitarra, flauta, clavecín, cello, etc. 
misma disposición interválica, los ejercicios y obras que estudian aspectos puntuales de la técnica (tremolo, tremolo en doble cuerda, digitación cromática, etc.) son interpretados con la misma digitación si se transportan un tono abajo. Félix de Santos $^{4}$ desarrolló una gran cantidad de obras para este instrumento, entre las cuales se destacan: Escuela moderna de la mandolina española, Escuela del tremolo op. 5, Doce estudios artísticos op. 17, Escuela del mecanismo, entre otras. Estas obras son fundamentales para el estudio de cualquier músico de plectro en España. El poder recopilar y sistematizar los procesos de enseñanza técnica (partituras) desembocó en la profesionalización y titulación de bandurristas en España, algo que en Colombia se viene afianzando hace aproximadamente 10 años.

"La bandola es hija legitima de la badurria" (Montoya,1988). ${ }^{5}$ No se sabe en que momento la bandurria llega a tierras americanas, se tiene referenciasde que fue traida por funcionaros de la corona española, y como otros instrumentos de corte popular (laud, guitarra, vihuela, etc.), no eran aceptados en ciertos círculos sociales.La bandurria era tradicionalmente utilizada en conjuntos de tunas o estudiantinas que interpretaban música popular española (jotas, flamencos, pasodobles, etc). Este aspecto popular interactuó con las músicas colombianas de la época, dando origen a otra palabra que incluyera ese aire nacional sin invocar a la palabra bandurria:

Según José Caicedo Rojas, la bandola o bandurria tenía en el siglo pasado un fondo curvo. Otros musicólogos dice que era de tamaño menor que la guitarra o de forma similar. El general Rafael Uribe Uribe, en su diccionario abreviado, ratifica lo anterior describiendo la bandola como un instrumento de música pequeño de cuatro cuerdas.(Marulanda y Gonzáles, 1994)

En esta época la bandola contaba con cuatro órdenes, más adelante los hermanos Amado empezaron a construir instrumentos con 5 órdenes:

Esta bandola data de pricipios de siglo $\mathrm{xx}$, es construdida por los hermanos Amado, reconocidos fabricantes de bandolas, tiples y guitarras, aquí es donde comienza la tranformacion del instrumento, ya que en ese momento cuenta con los dos primeros ordenes triplicados y los tres restante duplicados. (León, 1988) ${ }^{6}$

\footnotetext{
${ }^{4}$ (1874-1946), maestro de instrumentos de plectro del Conservatorio del Liceo de Barcelona.

${ }^{5}$ Diego Estrada Montoya (1937-2011), bandolista y compositor colombiano, comentario programa televisivo "música para todos, la bandola", 1988.

${ }^{6}$ Luis Fernando León, bandolista y compositor colombiano, comentario programa televisivo "música para todos, la bandola", 1988.
}

La melodias populares eran interpretadas en las nacientes bandolas, acompañadas de un nuevo intrumento, el tiple, de arraigo más papular, ya que al ser un intrumento armónico, satisfacia más las nececidades de los cantautores que la propia bandola:

Al contrariode lo que muchos creen la bandola nunca fue un instrumento campesino, fue un instrumento más urbano, no está vinculado con tradiciones campesinas a excepción de ciertos lugares en particular, siempre ha interactuado con músicas académicas por su origen urbano. $\left(\right.$ Bernal,2012) ${ }^{7}$

La bandola ha interactuado desde sus origenes con músicas académicas más que con lo popular, los primeros intentos por llevarla a las salas de concierto datan del año 1852 (todavía conservaba los 5 órdenes) en la llamada sociedad filarmónica. ${ }^{8}$

\section{Familia de las bandolas andinas colombianas}

Desde hace unos años elproceso de consolidacion de la familia de las bandolas andinas colombianas viene teniendo un desarrollo importante en el campo musical, con la aparicion de agrupaciones como la осв (Orquesta Colombiana de Bandolas), Cuarteto Perendengue, ${ }^{9}$ entre otras. Quienes han dado a conocer este formato en diferentes espacios de conciertos, foros, congresos y lugares de convocatorias de diferentes grupos musicales,apostándole a un repertorio variado y rico en ritmos y estilos interpretativos (música andina colombiana, latinomaericana, clásica académica, contemporánea y adaptaciones de música popular), ello ha creado el acercamiento de un nuevo público a este tipo de formatos inéditos hasta ahora. Tambien los constructores han realizado un gran aporte al interesarse por estas nuevas bandolas, las cuales están ahora en universidades y academias de Colombia para ser interpretadas dentro de formatos de música de cámara. Cabe destacar que la música andina colombiana es la raiz de la cual la bandola viene y que, aunque incorporemos otros repertorios, esta música es la columna vertebral de la idiocincracia de nuestros instrumentos y, por ende, es de la familia. No hay que desconocer tambien que los diferentes festivales de música andina colombiana han creado una especie de "temporada" de encuentros

\footnotetext{
${ }^{7}$ Manuel Bernal, bandolista, profesor de bandola, Academia Superior de Artes de Bogotá, comentario nota periodística "cuerdas más cuerdas", el tiempo.com

${ }^{8}$ (1846-1857) Fundada por Henry Prince (1819-1863), fue la primera institución que se creó en Bogotá para la enseñanza de la música, la cual a su vez estuvo vinculada con la mayoría de la actividad musical del país.

${ }^{9}$ Cuarteto clásico de bandolas. Es una agrupación bogotana integrada por 2 bandolas sopranos, 1 contralto y 1 barítono-tenor. Interpretan música andina colombiana, latinoamericana, contemporánea y académica clásica. Actualmente tienen un trabajo discográfico.
} 
alrededor de esta música, pero los espacios en las universidades y, más especificamente, con los bandolistas es limitado, ya que muchos interpretan como ya lo hemos mencionado, otro tipo de repertorios o repertorios andinos colombianos de compositores contemporáneos o modernos, y la escuela interpretativa de grandes bandolistas o compositores clásicos de la música colombiana queda en el ol vido.

\section{Bandola andina colombiana (soprano)}

La bandola andina colombiana es un instrumento soprano de cuerdas pulsadas y mástil trasteado, de la familia de las bandolas andinas colombianas. En la actualidad posee 12 cuerdas afinadas en 6 órdenes dobles, aunque en las primeras etapas de su desarrollo contaba con 16 o 14 cuerdas en órdenes triples y dobles. Se afina en $440 \mathrm{~Hz}^{10} \mathrm{y}$ en la tonalidad de do, aunque también en los primeros años se afinaba en si bemol. Se ejecuta tañendo las cuerdas con un plectro, ${ }^{11}$ lo que la convierte en pariente de instrumentos ejecutados de la misma forma como, la mandolina, la bandurria, el bandolín, etc. La bandola es utilizada para ejecutar cualquier tipo de melodía ya sea de música académica, religiosa o popular, pero desde su aparición en el siglo XIX se ha utilizado para interpretar música andina colombiana, ${ }^{12}$ lo cual la convirtió en referente para dicha música.

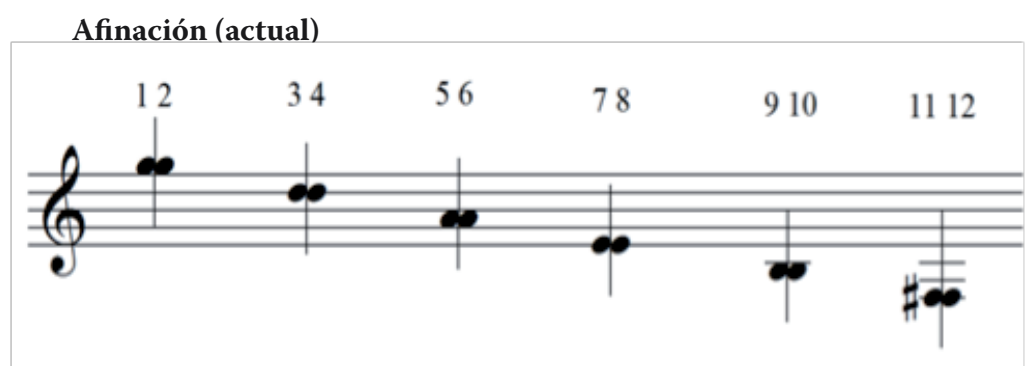

Características de los registros

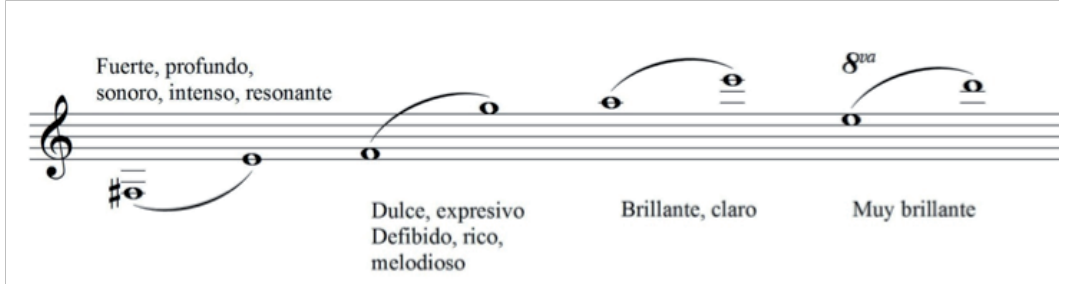

10 Patrón estándar para la afinación musical.

${ }^{11}$ Uña, pajuela o púa hecha generalmente en forma triangular de distintos materiales (tortex, Nylon, celuloide, lexan), el cual sirve para ejecutar instrumentos de cuerda, el más común guitarra eléctrica

${ }^{12}$ Música tradicional de los departamentos de la zona andina de Colombia.

\section{Movimientos fundamentales}

Se han sistematizado cuatro movimientos fundamentales para el desarrollo de una buena técnica del instrumento, ${ }^{13}$ esto con base en un sonido pastoso, redondo y con proyección, que garantice la calidad de las ejecuciones en los diversos estilos musicales. Una de las apuestas con estos movimientos es que la bandola gane un sonido de base y que este sea el punto de partida para buscar un sonido personal, como cualquier músico o instrumentista:

Yo si soy un convencido de que hay que desarrollar una sonoridad general de base del instrumento, de ahí en adelante construir el sonido de uno, no quiero hablar de que se vuelva un estereotipo de que todo el mundo toque igual, no para nada, eso me parece desagradable, pero lo que sí creo como docente del instrumento, me preocupo por trabajar, es que uno debe consolidar un sonido básico, todo el mundo debía tenerlo, como ocurre con el violín, con la guitarra o ese tipo de cosas de ahí para arriba uno construye su sonido que deriva de experiencias personales, del gusto del carácter de tanta cosas.(Forero, entrevista, 2013)

\section{Técnicas de ejecución de la bandola andina colombiana}

La siguiente clasificación de técnicas tiene por objetivo mostrar los diferentes aspectos que de una $u$ otra manera se han venido incorporando a la bandola andina colombiana durante su historia, ya que si bien algunas de estas técnicas no era utilizadas por todos los bandolistas en todas las épocas, sí fue gracias a su experiencia y domino del instrumento que se fueron consolidando hasta ser parte del instrumento de una manera natural y autónoma. Ya que es bueno recordar que el estudio de la bandola va ligado en muchas ocasiones a la tradición oral o a espacios distintitos a la academia, que son la connotación de ser un instrumento venido y nacido de las músicas populares.

${ }^{13}$ Se tiene registro de métodos que hablan de la forma de coger la bandola, coger el plectro, sentarse y atacar las cuerdas, uno de los más famosos es el del bandolista Diego Estrada. Pero ninguno habla de una técnica que vaya en pro de un sonido del instrumento. 
Técnicas tradicionales de ejecución ${ }^{14}$

Plectro abajo-arriba ${ }^{15}$

Significa la dirección del ataque del plectro:

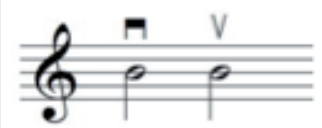

Alza púa

Es la union de dar un golpe hacia abajo y uno hacia arriba

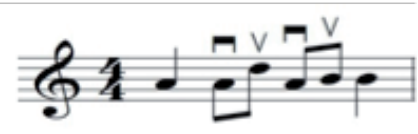

Contra púa
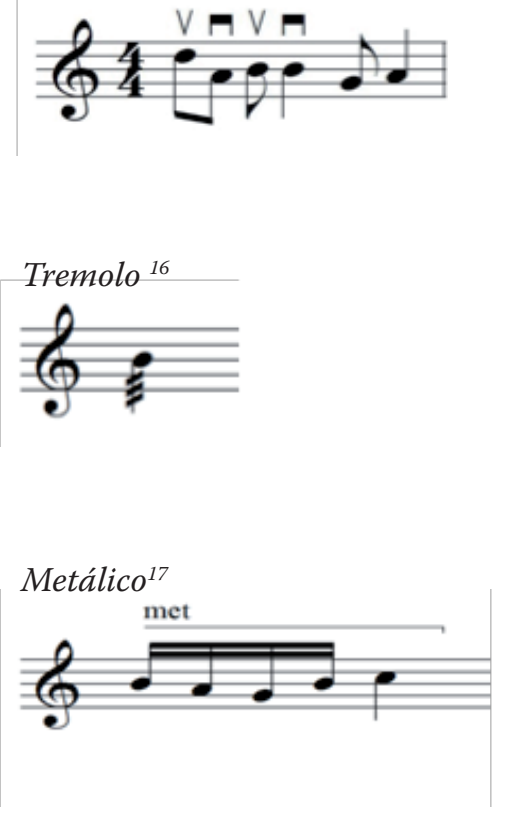

${ }^{14}$ Las técnicas ejecución ha estado ligadas a la actitud interpretativa del bandolista a lo largo de la historia. La forma de coger el plectro, hacer digitaciones, escalas, interpretar pasajes rápidos, han sido logradas por vocación más que por una escuela que sistematice estos aspectos. Aunque sí se puede hablar de algunos estilos y formas de solucionar estas cuestiones técnicas de la bandola que crearon algunos intérpretes.

15 Se utiliza la misma grafía que para los instrumentos de cuerda frotada, significando lo mismo pero con el plectro. Instrumentos con más tradición académica como la mandolina y la bandurria lo han utilizado por mucho tiempo.

${ }^{16}$ El tremolo también es utilizado en otros instrumentos (cuerda, percusión, viento, teclado).

17 En los instrumentos de cuerda frotada este efecto se le suele llamar sulponticello (cerca del puente)
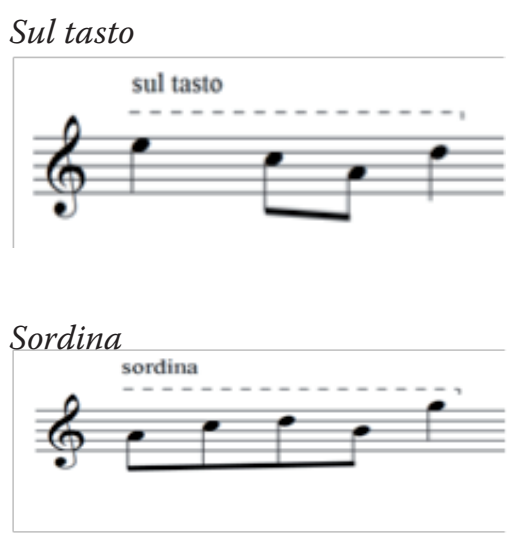

\section{Técnicas extendidas}

Las técnicas extendidas de un instrumento son el conjunto de nuevas formas, tanto técnicas como expresivas, que no son históricamente las utilizadas en la ejecución tradicional. Estas técnicas buscan encontrar nuevos sonidos, "ruidos", timbres o efectos, los cuales son utilizados en diferentes obras. En los instrumentos con gran tradición histórica de ejecución como el caso del violín, flauta, piano, guitarra, clarinete, entre otros; estas técnicas han aparecido en los últimos 60 o 70 años, más específicamente con el rompimiento del periodo de la práctica común ${ }^{18}$. Aunque estas técnicas han estado ligadas a la música contemporánea, son utilizadas también en repertorios tradicionales, para dar variedad y enriquecer a la obra y al intérprete.

Percutidos: Son aquellas que se realizan golpeando diferentes zonas de la bandola (algunas con y sin las cuerdas). Se pueden realizar con cualquiera de las 2 manos o utilizando objetos de diferente material, en el caso de la bandola la utilización del plectro para hacer percutir, también es una opción muy cómoda. El realizar las percusiones se debe tener en cuenta el timbre o el color que se quiere obtener, ya que dependiendo dela zona de la bandola esto cambia.

\footnotetext{
${ }^{18}$ Generalmente se comprende este periodo en la historia de la música occidental que va desde (1600-1900) en el cual la práctica armónica se basaba en un centro tonal o función tonal, la cual subordina a sus diferentes grados de su escala diatónica, este fenómeno de relación-subordinación se llama tonalidad.
} 


\section{Efectos sobre cuerdas}

Se realizan haciendo distintos movimientos, ya sea con la mano izquierda o el plectro

Algunos de ellos son:

Pizzicato Bartok ${ }^{19}$ o slap pizzicato: Este pizzicato es particularmente fuerte y se logra haciendo rebotar la cuerda contra el diapasón del instrumento, en el caso de la Bandola Andina colombiana este se ayudará con un movimiento especial del plectro.

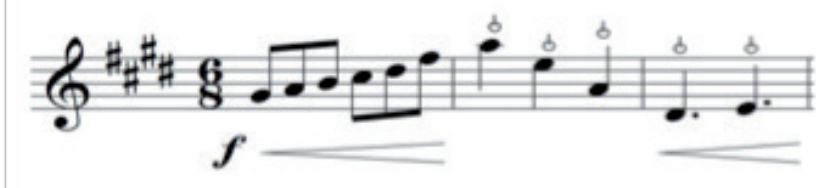

Glissandos: ${ }^{20}$ Este efecto consiste en pasar rápidamente de un sonido a otro, haciendo que se escuchen todos los sonidos intermedios en lo posible; en el caso de instrumentos de cuerda como la bandola, este se hace deslizando sobre la cuerda con el mismo dedo. Hay diferentes maneras de matizarlo ya sea con la intensidad o la velocidad:

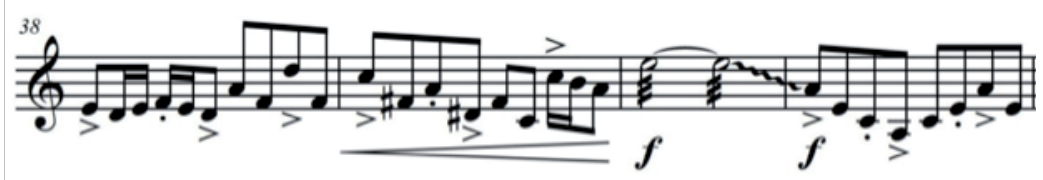

En algunas partituras se encuentra la línea del gráfico anterior, la cual está formada con ondas.

Vibratos verticales: Este efecto se produce realizando un movimiento vertical sobre la cuerda produciendo un sonido oscilante de la nota tocada. Hay diferentes maneras de matizarlo ya sea en la intensidad o en la velocidad del vibrato: "Se indica con una o dos flechas en direcciones opuestas, la líneas onduladas señala el número de veces que se desplaza el dedo (verticalmente). Se puede hacer doble, triple y cuádruple" (Torres, 2010).

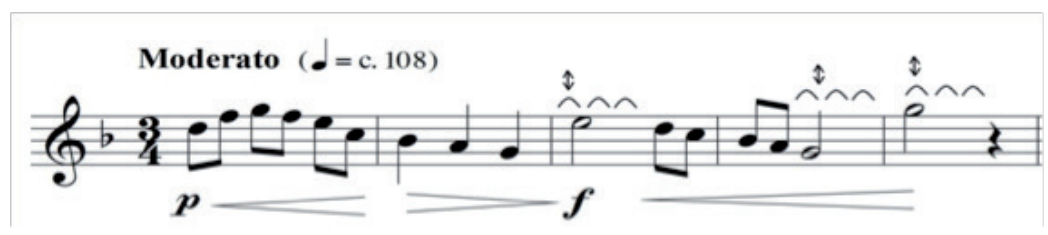

Arpa I y II: Este efecto se logra atacando las cuerdas (plectro, mano derecha o izquierda) detrás del puente o detrás del hueso de la cabeza, ${ }^{21}$ tiene una calidad brillante, resonante y con variedad de matices.

\footnotetext{
${ }^{19}$ Se le dice Bartok, ya que Bela Bartok (1881-1945), compositor húngaro, lo utilizaba muy a menudo dentro de sus composiciones

${ }^{20}$ Cabe destacar que los glissandos se pueden complementar con técnicas tradicionales como el tremolo y el tremolo en doble cuerda, como también con acordes

${ }^{21}$ Esta técnica está tanto en las percutidas como en las de efectos de cuerdas.
}

Rasgueos $^{22}$ : Se realizan con movimientos rápidos arriba y abajo (generalmente sobre las cuerdas) ${ }^{23}$, se pueden utilizar distintos materiales para hacer el rasgueo pero lo más natural será con el plectro, a menos que se quiera un efecto en especial. Se indicará con una flecha la dirección del rasgueo, aunque en algunos casos las direcciones de los rasgueos serán a criterio del intérprete. Para los rasgueos se debe tener en cuenta el ritmo, el efecto y la dinámica que se desee, ya que hay un sin número de combinaciones rítmicas.

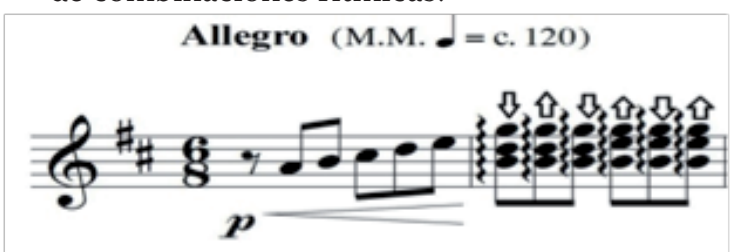

Para los rasgueos que sean con las cuerdas tapadas solamente se indicará el ritmo y la abreviatura (Ras. Tap.) se escribirá sobre las notas de las cuerdas al aire que serán tocadas, las cuerdas se taparan con la mano izquierda. En el caso de notas pisadas y que se debe levantar los dedos de la mano izquierda se realizará el mismo procedimiento.

\section{Técnicas complementarias}

Son el conjunto de técnicas que tradicionalmente se utilizan en determinados instrumentos (violín, guitarra, piano, flauta, etc.), pero adaptadas para su ejecución en otro ${ }^{24}$. En instrumentos como la bandola andina colombiana, en la cual su formación tanto técnica como académica está en construcción, las técnicas tradicionales van de la mano de los intérpretes y han sido enseñadas mediante tradición oral o imitación, en algunos casos se han tratado de escribir en partituras (grafías)

\footnotetext{
${ }^{22}$ Para los rasgueos se debe tener en cuenta el ritmo, el efecto y la dinámica que se desee, ya que hay infinidad de ritmos nos dedicaremos a explicar cómo se hacen y no unos rasgueos predeterminados.

${ }^{23}$ También se pueden hacer rasgueos en las cuerdas que se encuentran detrás del puente o detrás del hueso de la cabeza.

${ }^{24}$ En la historia de la música, se han visto casos en los que algunos instrumentistas tratan de emular las técnicas de otros instrumentos.
} 
tomando elementos de otros instrumentos de cuerda y no exclusivamente de plectro $^{25}$, algunos de ellos son del violín y la guitarra ${ }^{26}$ :

Es muy importante sistematizar las técnicas que se pueden emplear de otros instrumentos de cuerda, algunas son utilizadas por la mandolina, la domra y la bandurria, pero en el caso de la bandola por su afinación y timbre, estas técnicas darían un resultado distinto, y se volverían parte de la técnica de la bandola andina colombiana.

Estas técnicas complementarias son un campo nuevo de exploración con unas posibilidades ilimitadas para su uso, y aunque algunas técnicas (extendidas y complementarias) ya son empleadas por otros instrumentos, estas ayudarán al músico-bandolista a generar un criterio interpretativo y compositivo para el instrumento. A continuación sistematizaremos las técnicas utilizadas para este trabajo y el instrumento en el cual son utilizadas.

Pizzicato de mano izquierda (violín): Este efecto se realiza al pulsar las notas indicadas con los dedos de la mano izquierda, se utiliza una cruz como símbolo de este efecto $(+)$.

Cuando se ha marcado una cruz encima de unanota, la cuerda se toca con uno de los dedos de la mano izquierda. A menudo, estas soncuerdas al aire y se tocan con el meñique. Otras veces se debe tocar con la mano izquierda una serie de sonidos. En este caso, el dedo se usará para producir el sonido más agudo, pulsa el próximo sonido más agudo; así sucesivamente. (Adler, 2006)

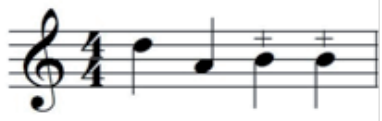

Sweeppicking o barrido (guitarra eléctrica): Es un efecto o movimiento, en el cual se tocan una sucesión de notas con mucha rapidez sobre cuerdas consecutivas con el mismo movimiento del plectro (abajo-arriba), fue popularizada en los años 70 por los guitarristas eléctricos para imitar movimientos del arco del violín y poder interpretar piezas del periodo clásico. ${ }^{27}$

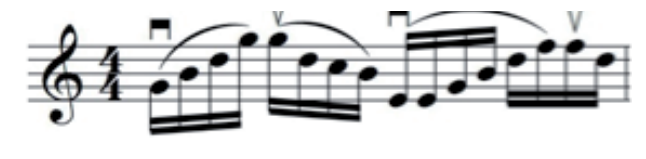

\footnotetext{
${ }^{25}$ Es curioso, pero en el caso de los instrumentos de cuerda pulsada los bandolistas han tenido un contacto casi nulo en cuanto a información sobre instrumentos de plectro se refiere. Solo hasta hace poco se comenzó a intercambiar conocimientos con instrumentos como la bandurria, la mandolina y el bandolín, a través de encuentros, seminarios y talleres.

${ }^{26}$ Véase el apartado "Técnicas tradiciones de ejecución”, en este mismo artículo.

${ }^{27}$ Estos movimientos en la guitarra eléctrica se hacen ligando algunas notas con la mano izquierda, en la bandola andina son muy pocas la notas que se pueden ligar de esta forma, es por eso que casi todas la notas deben articularse, siendo esto, un factor determinante en el uso de esta técnica.
}

Lija (violín): Efecto realizado al raspar las cuerdas del violín detrás del puente, se obtiene un sonido parecido a una lija o una chicharra. Astor Piazzolla popularizó este efecto en sus composiciones de tango contemporáneo y en sus diferentes conjuntos instrumentales. Cabe anotar que no se encontró una grafía para este efecto, pero sí están la grabaciones en las que se utiliza. ${ }^{28}$ La grafía utilizada será la de un símbolo de (+) en la segunda línea (sol), el efecto se consigue haciendo el movimiento sobre la sexta cuerda o la sexta cuerda en el área (arpa II). Se debe "raspar" la cuerda con el plectro en forma diagonal a esta, con el borde del plectro.

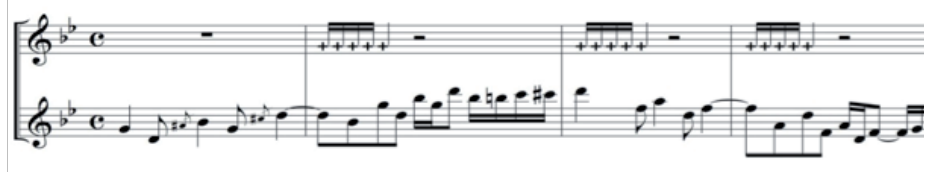

También se puede hacer un efecto a manera de glissando de lija, sobre la sexta cuerda (arpa II), o se logra haciendo la misma técnica anterior, pero en una sola dirección.

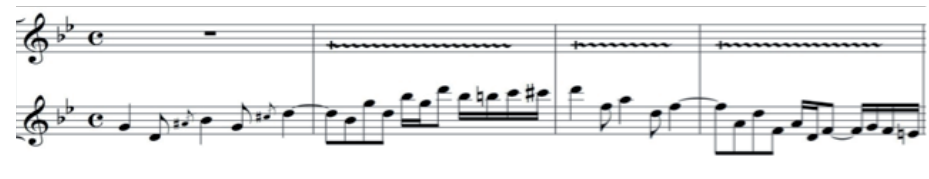

Scordatura: Término italiano que significa "afinación incorrecta", es utilizado por los instrumentos de cuerda para crear ciertos efectos de color y facilidad técnica en algunos pasajes, se considera scordatura cuando se altera la afinación tradicional del instrumento:"La afinación en scordatura se utiliza desde el siglo XVII para facilitar la interpretación de fragmentos difíciles en tonalidades remotas, para obtener acordes poco corrientes y para cambiar el color tonal del instrumento"(Adler, 2006).Cabe anotar que el compositor debe indicar previamente la afinación de las cuerdas que desee, ya sea al principio de la partitura o en la parte que se vaya a utilizar.

\section{Explicación de la signografía}

Algunos de los signos empleados en las técnicas complementarias fueron tomados directamente de los instrumentos a los cuales pertenecía dicha técnica, en algunos casos específicos no se pudo encontrar una signografía propia y se tuvo que crear. En el caso de las técnicas extendidas, aquellas que fueron hechas para la bandola, los signos fueron creados por quien escribe.

Se hizo una clasificación de 13 áreas divididas en 2 zonas. Los siguientes ejercicios se deben realizar en todas las13 áreas, algunas ofrecerán mayor variedad tímbrica y otras, efectos propios de esa área. Cabe anotar que se le dará una altura en los sonidos del pentagrama a cada área para diferenciarlas, ya que se utilizará el símbolo de percusión

\footnotetext{
${ }^{28}$ En el siguiente enlace el violinista Fernando Suarez Paz explica cómo se realiza este efecto http://www.youtube.com/ watch? $=$ rNwsnSIA0WQ
} 
tradicional (x), sin embargo para el área 7 (boca), y las arpas (arpa I y arpa II) se hará un tratamiento especial, ya que en el caso de la boca puede percutir sonidos con alturas definidas, por lo que se explicará aparte su grafía. Con respecto a las arpas, funcionan también en la categoría de efectos en cuerdas, por lo que se hará una grafía diferente cuando se utiliza en este contexto, por ahora se utilizará como las demás.

Nomencaltura de las manos:

Mano Izquierda: (MI)

Manos Derecha. (MD)

\section{Zonas}

\section{Zona $A^{29}$}

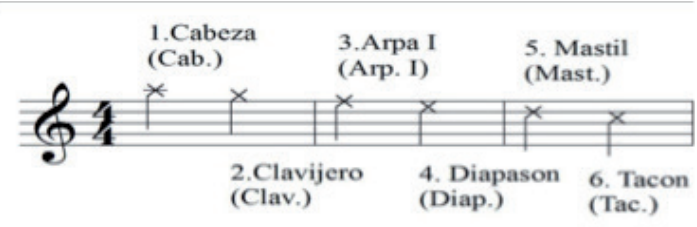

\section{Zona B}
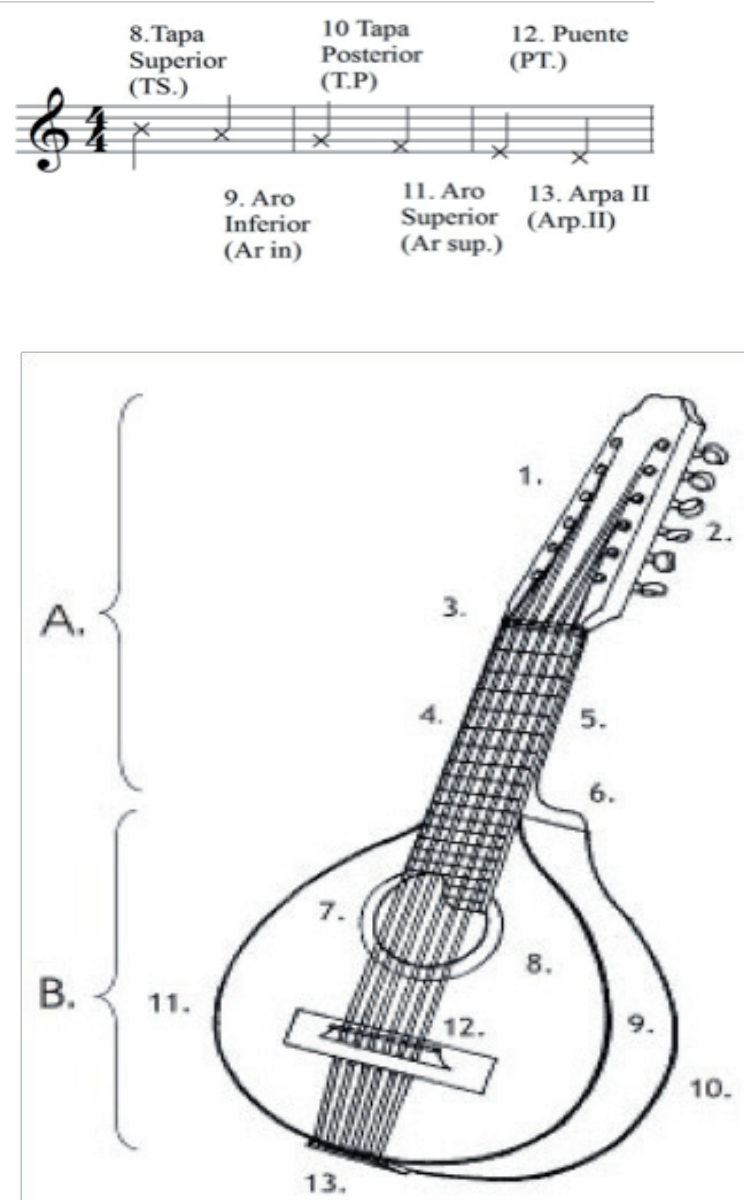

\footnotetext{
${ }^{29}$ Los diminutivos se escribirán siempre arriba del símbolo en la partitura, en este caso se escribieron uno abajo y uno arriba por la disposición del espacio.
}

\section{Características de las zonas:}

El instrumento será dividido en 13 áreas repartidas en 2 zonas. $^{30}$

\section{Zona A}

1. Cabeza: Poca resonancia, poco sonido.

2. Clavijero: Poca resonancia, poco sonido

3. Arpa I: Brillante, poco resonante.

4. Diapasón: Resonante, con variedad de matices.

5. Mástil: Efectos de carácter seco y resonante.

6. Tacón: Efectos de carácter seco, poco resonante, pocos matices.

\section{Zona B}

7. Boca: Efectos resonantes, variedad de matices.

8. Tapa superior: Variedad tímbrica (se puede percutir con los dedos, las uñas, dando otro timbre) resonante, definido, variedad de matices.

9. Aro inferior: Variedad tímbrica, seco, poco resonante, variedad de matices.

10. Tapa posterior: Variedad tímbrica, oscuro, resonante, variedad de matices.

11. Aro superior: Variedad tímbrica, seco, poco resonante, variedad de matices.

12. Puente: Seco, resonante, variedad de matices.

13. Arpa II: Brillante, poco resonante, poco sonido.

\section{Ejercicios preparatorios}

Los siguientes ejercicios fueron creados para ser utilizadoscomo preparatorios para las obras que seguirán al siguiente capítulo, cada uno busca desarrollar una técnica o efecto preciso y el detallado trabajo del musico-bandolista será fundamental para esto, ya que no solo es un ejercicio técnico, sino tambien creativo, que busca una interacción en entre la técnica y la música.

\footnotetext{
${ }^{30}$ Las grafías para las áreas será explicada en la propuesta metodológica.
} 


\section{Ejercicio 14}

Se recomienda exagerar las dinámicas, ya que estos efectos con las arpas crean atmóferas que son muy útilies en fraseos contundentes y que combinados con notas reales, en este caso acordes, dan colores particulares a estas notas. Se pueden hacer variaciones de todo tipo, lo escencial es combinar el sonido particular de las arpas con lo que se desee dentro del ejecicio ${ }^{31}$.

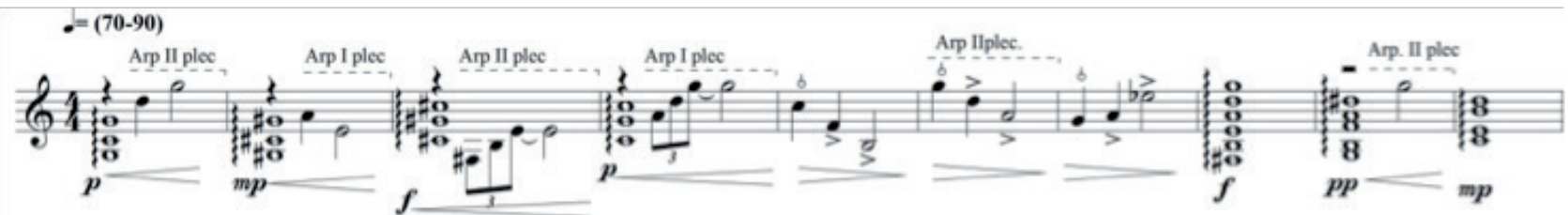

\section{Cuatro obras para bandola sola}

Se compusieron cuatro obras para efectos pedagógicos dentro del trabajo monográfico: "Un sueño", "Pantaleón", “Odeón” y "Bandolarium”, las cuales vienen con la partitura respectiva y se puede consultar en una descripción de la obra, qué técnicas se utilizan y algunas recomendaciones de interpretación. Como se mencionó anteriormente estas obras utilizan técnicas tradicionales de ejecución con las nuevas técnicas logrando una fusión para el músico-bandolista.

"Un sueño", en este fragmento se muestra la técnica del SweepPicking:

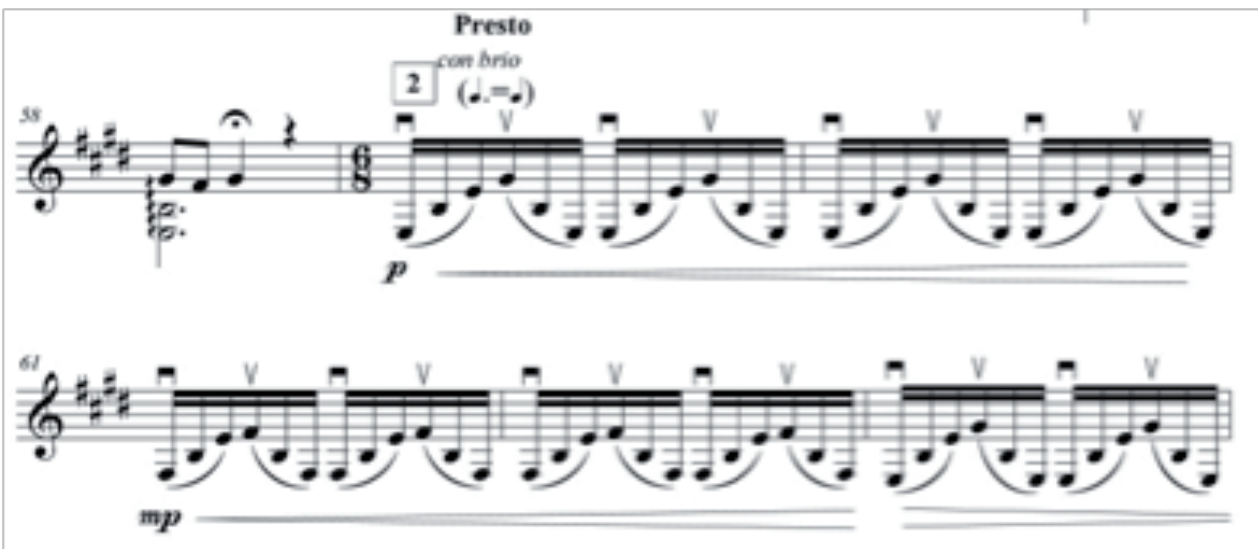

“Pantaleón”, en este fragmento se muestra las técnicas de percusión, arpas y rasgueo:

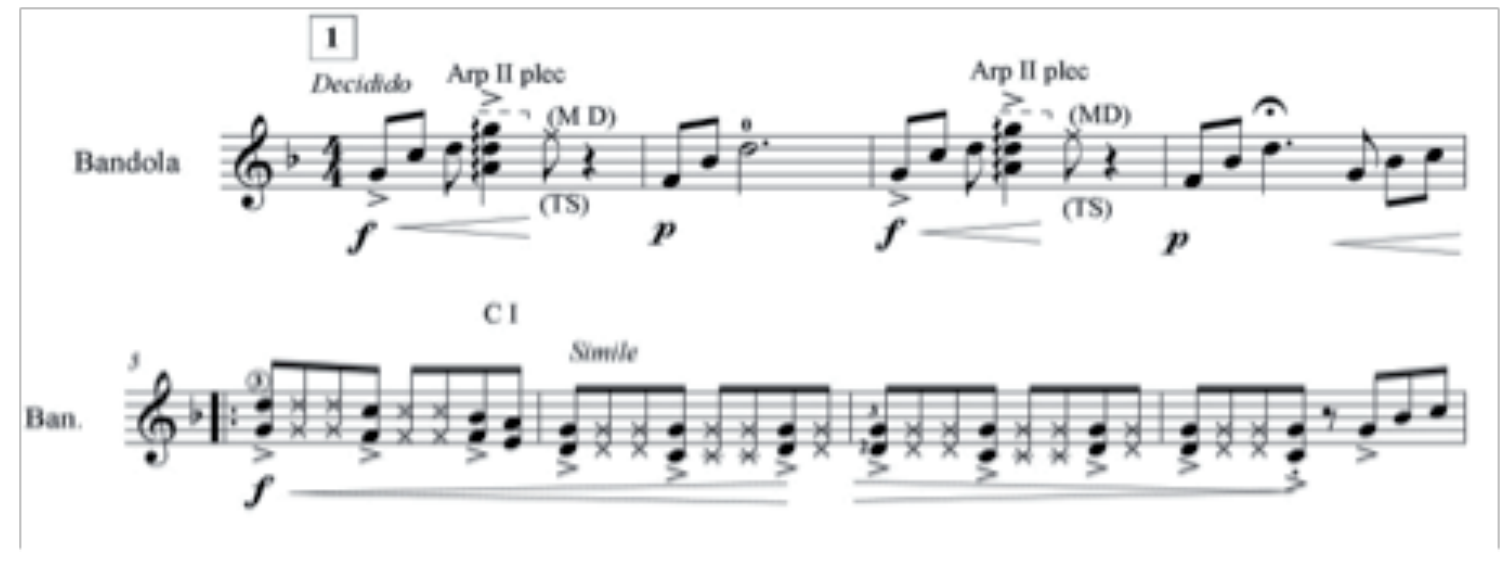

\footnotetext{
${ }^{31}$ Es importante anotar que al tratarse de cuerdas en las áreas de las arpas, se pueden utilizar técnicas tradicionales, por ejemplo el tremolo, metálico, etc.
} 
“Odeón”, en este fragmento se muestra técnicas de percusión sobre varias zonas de la bandola:

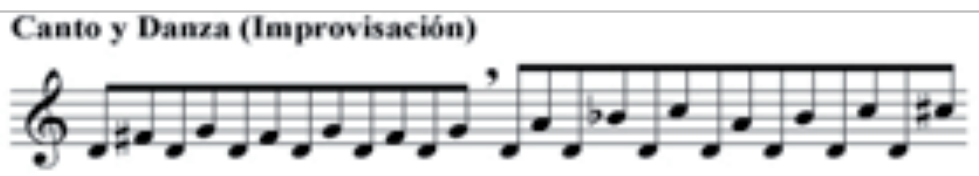

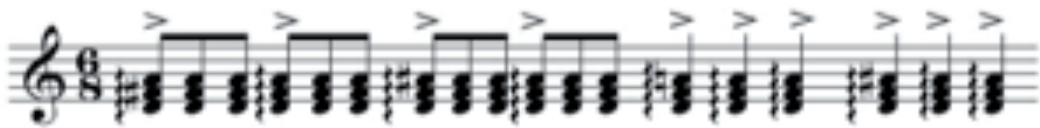

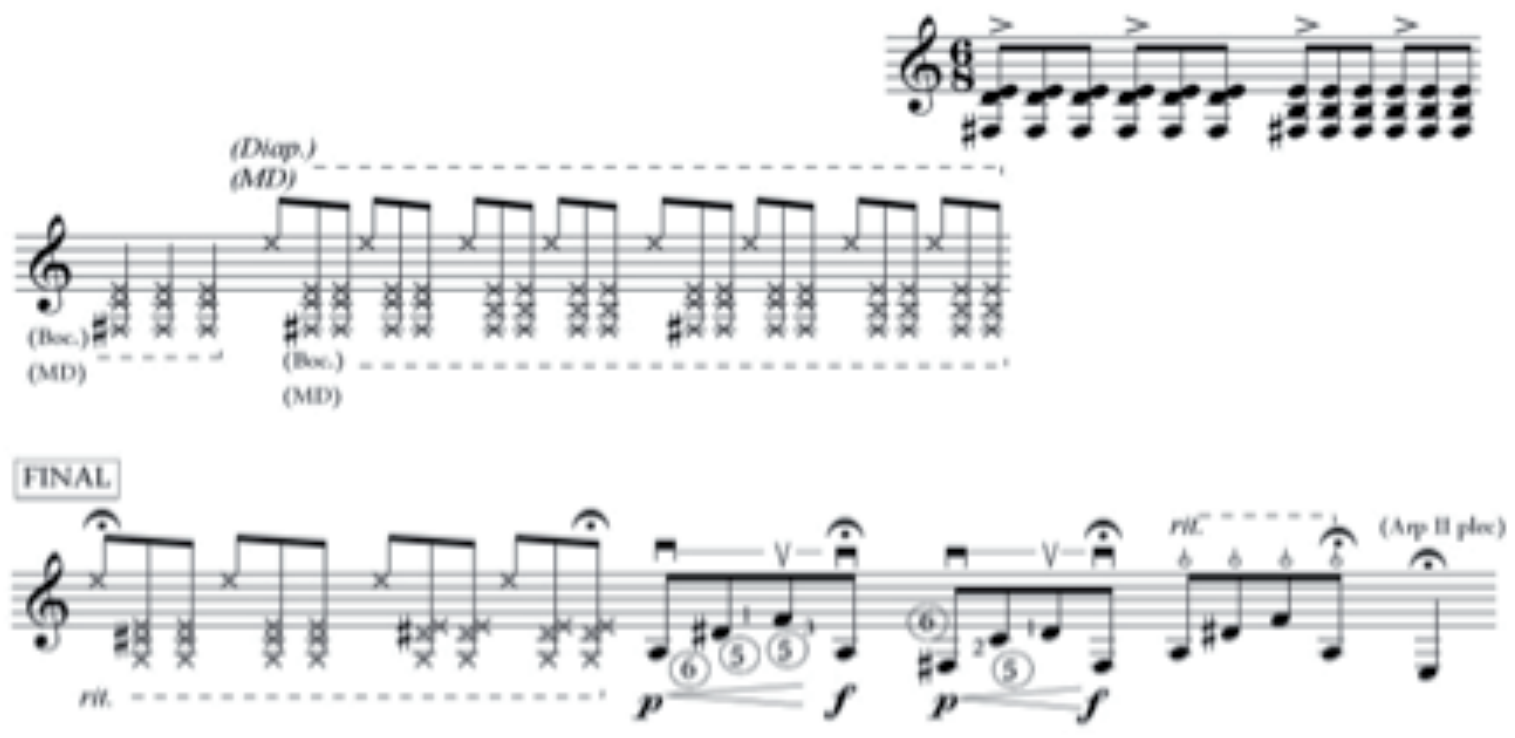

"Bandolarium", en este fragmento se observa la utilización del pizzicato de mano izquierda utilizado en el violín:

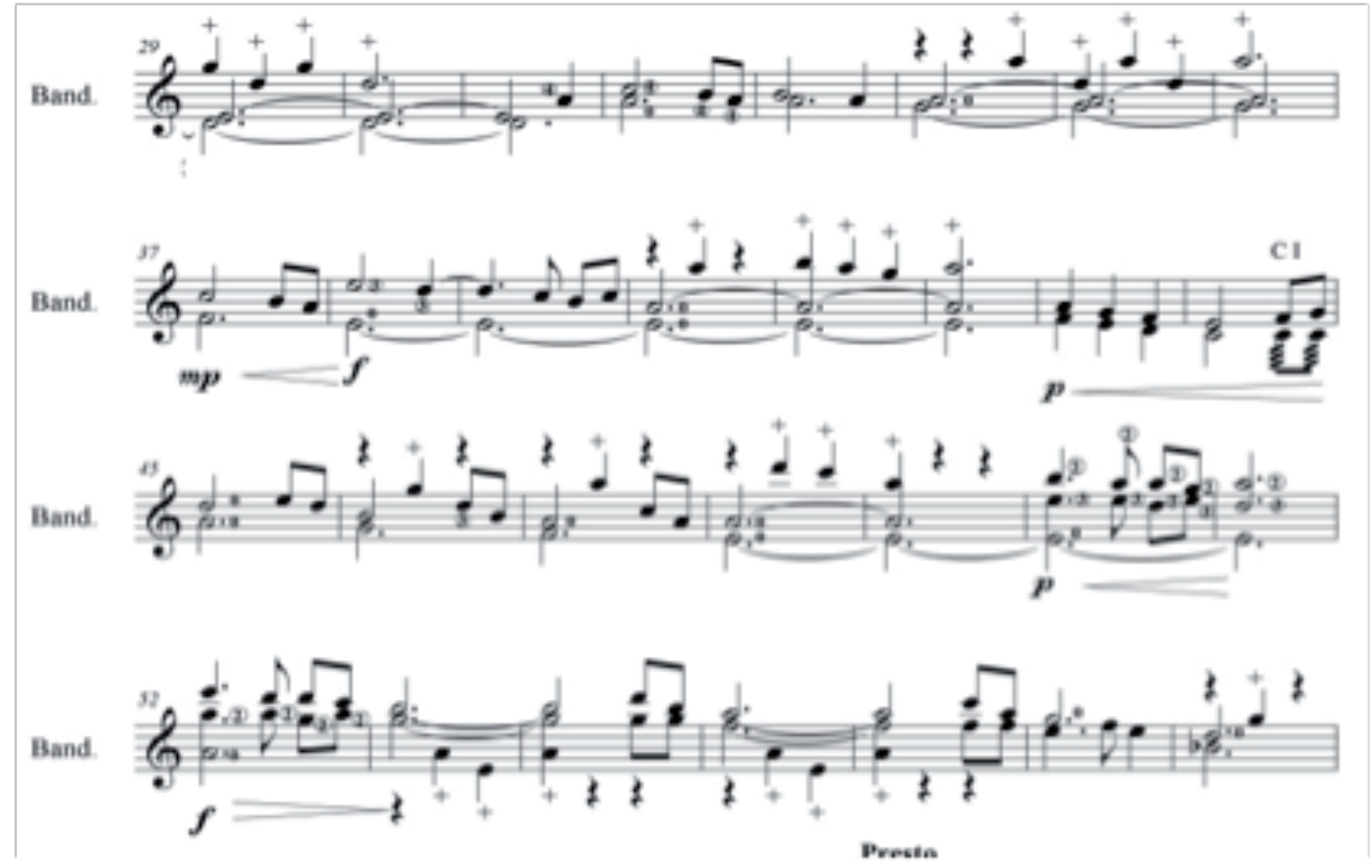




\section{Referencias bibliográficas}

Adler, S. (2005). El estudio de la orquestación. Madrid: Ideabooks.

Bernal, Manuel. (2003).Cuerdas más cuerdas menos, una visión del desarrollo morfológico de la bandola andina colombiana [Monografía para optar por el título de licenciado en música].Universidad Pedagógica Nacional, Facultad de Bellas Artes, Bogotá.

Canal EL Tiempo. (2012).Cuerdas más[ programa televisivo]. Bogotá.

Forero, F. (2011). Entre cuerdas y recuerdos. Bogotá: Sic ediciones.Marulanda, O., y Gonzáles, G. (1994). Pedro Morales Pino, la gloria recobrada. Ginebra (Valle), Colombia: Funmúsica.

Orquesta filarmónica de Bogotá (1988).Música para todos, la bandola [Programa de televisión]. Bogotá.

Royo, A. (2000). El análisis como herramienta de interpretación de la música atonal para guitarra. Revista electrónica Léeme. Disponible en: http://musica.rediris.es/ leeme/revista/royo06.pdf

Torres, V. (2010). Piel de guitarra [monografía de grado]. Universidad Pedagógica Nacional, Bogotá.

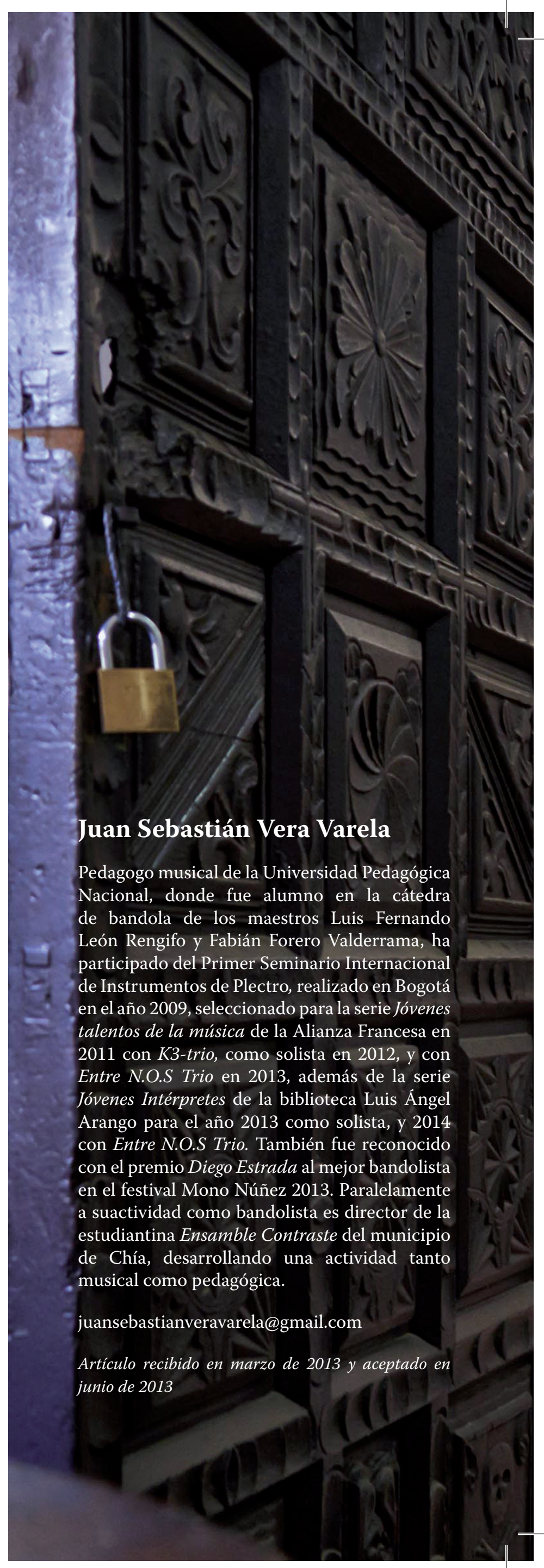


